\title{
MALÁRIA INDUZIDA NO ESTADO DE SÃO PAULO, BRASIL.
}

\author{
José Carlos Rehder de Andrade e Dalva Marli Valério Wanderley
}

\begin{abstract}
Procedeu-se uma revisão dos 58 casos de malária induzida diagnosticados em São Paulo, de janeiro de 1983 a julho de 1990 , correspondendo a $0,3 \%$ do total de casos de malária confirmados. As variáveis sexo, idade, nível de parasitemia, sintomatologia, evolução e tempo médio de início dos sintomas e o diagnóstico foram analisadas entre os 25 casos infectados por transfusão e 28 por uso de agulhas contaminadas. Predominou a infecção por Plasmodiun vivax $(79,4 \%)$, destacando-se $5,6 \%$ de casos com infecção mista. A idade média dos infectados por uso de agulhas foi significativamente menor $(\mathrm{p}<0,005)$ do que entre os infectados por transfusão. O tempo médio entre o início dos sintomas e a confirmação do diagnóstico foi maior entre os infectados por agulha do que entre os por transfusão, embora a diferença não tenha sido estatisticamente significante $(p>0,10)$. Apesar dos casos induzidos representarem percentual pequeno em relação ao total de casos notificados e a transmissão através do sangue estar sob controle, a transmissão acidental por agulhas contaminadas devido ao uso de drogas injetáveis, constitui risco para o restabelecimento da malária em áreas do Estado de São Paulo.
\end{abstract}

Palavras-chaves: Malária. Epidemiologia. Malária transfusional. Malária entre toxicômanos.

No Estado de São Paulo, a transmissão da malária foi controlada em meados da década de 60. Desde então, os casos diagnosticados têm sido investigados epidemiologicamente com vistas a caracterizar o momento, o espaço e a forma de transmissão. Informações da Superintendência de Controle de Endemias (SUCEN), demonstram que cerca de $90 \%$ dos casos são importados da área endêmica de malária brasileira, a região amazônica ${ }^{10}$.

A partir de 1983 a vigilância epidemiológica da malária foi intensificada no Estado, com vistas a detectar e tratar precocemente as fontes de infecção, evitando assim a instalação de surtos de transmissão ${ }^{11}$ tendo em vista o aumento da incidência no país ${ }^{9}$. A par deste

Superintendência de Controle de Endemias - SUCEN da Secretaria de Estado da Saúde do Estado de São Paulo.

Endereço para correspondência: Dr. José Carlos Rehder de Andrade. SUCEN, R Paula Souza 166 Luz, 01027 São Paulo, SP, Brasil.

Recebido para publicação em 14/05/91. trabalho, relata-se uma autoctonia média de 2,0\% nos últimos anos. Ao lado da quase inexistência de transmissão local por anofelinos vetores, tem sido observada anualmente a ocorrência de casos induzidos de malária, classificados na categoria de malária transfusional's . Casos esporádicos de malária congênita e outros classificados como mecanismo de transmissão excepcional também foram confirmados. Mais recentemente, a partir de 1987 , tem sido verificada a transmissão de malária acidental por agulha devido a inrtrodução não intencional de parasitas através de instrumentos ou seringas contaminadas?.

Se por um lado a estratégia de controle da malária para os casos de transmissão natural está bem determinada e sua aplicação tem significado êxito no sentido de conduzir à extinção dos focos de transmissão ${ }^{1}$, por outro lado, o controle da malária induzida ainda necessita ser estruturado no sentido de evitar a disseminação, não só da doença como também de outras patologias. O presente trabalho apresenta uma revisão dos casos induzidos de malária diagnosticados no Estado de São Paulo, 
no período de janeiro de 1983 a julho de 1990 , com vistas a fornecer subsídios para o seu controle.

\section{MATERIAL E MÉTODOS}

Foram estudadas 58 notificações de malária induzida, dentre as 17.440 investigações epidemiológicas realizadas, no período de janeito de 1983 a julho de 1990 . Os casos confirmados pela técnica da gota espessa, foram classificadòs segundo o mecanismo de infecção proposto pệr Bruce-Chwatt ${ }^{5}$, em: 1) malária transfusional relacionada com terapia envolvendo transferência de sangue total ou seus componentes; 2) malária acidental por agulha devido à introdução não intencional de parasitas por instrumentos ou seringas contaminadas; 3) malária congênita, na qual um recém-nascido é infectado através de placenta ou da passagem de parasitas da mãe e 4) malária excepcional com transmissão de plasmódios por malarioterapia ou acidentes de laboratório.

Procurou-se avaliar al gumas variáveis dos indivíduos infectados, como sexo, idade, nível de parasitemia, sintomatologia, evolução de caso, e tempo médio entre o início dos sintomăs/ diagnóstico, comparando-se o grupo dos infectatos por transfusão de sangue e o grupo dos infectados por uso de agulhas contaminadas. Para a comparação de alguns valores utilizou-se testes je significância paramétricos ( $\mathrm{t}$-Student).

\section{RESULTADOS}

A distribuição dos 58 casos segund o mecanismo de infecção (Tabela 1) aponta para 28 $(48,3 \%)$ transmitidos por agulha contaminada, 25 $(43,1 \%)$ por transfusão de sangue, $3(5,2 \%)$ com transmissão excepcional e $2(3,4 \%)$ de fo congênita. A espécie de plasmódio predominañte foi Plasmodium vivax em $79,4 \%$ dos castos, destacando-se $8,6 \%$ de casos com infecção mista por $P$. vivax e $P$. falciparum. Nota-se a ocorrência de malária por transfusão de sangue em todos os anos, destacando-se no ano de 1984 o registro de 6 casos na Grande São Paulo, sendo 5 de maláriá mista ( $P$. vivax e $P$. falciparum) e 1 por $P$. vivax. As investigações epidemiológicas destes casos revelaram que todos haviam doado sangue num único serviço de hemoterapia e que, durante o processo de doação, haviam sido submetidos a procedimento de plasmaferese. Um paciente com infecção mista evoluiu para o óbito. $O$ intervalo entre o início dos sintomas e o diagnóstico foi em média de 34,5 dias (11 a 76 dias). Através dos dados obtidos a partir das entrevistas com os pacientes, foi possível concluir pela existência de uma fonte única de infecção para os 6 casos $^{2}$.

No que diz respeito à transmissão por agulha contaminada, cabe ressaltar dois espisódios detectados nas cidades de Presidente Prudente e Bauru. O primeiro deles, em Presidente Prudente, iniciado em 1988 totalizou 12 casos. A investigação detalhada desta transmissão ${ }^{7}$ apontou a fonte de infecção importada de Mato Grosso, que iniciou a disseminação da malária entre indivíduos que faziam uso de drogas em grupo. Entre os casos, dois pacientes apresentaram reinfecções, isto é, tiveram novo acesso malárico após o tratamento com cloroquina e primaquina. Testes sorológicos para HIV em 6 pacientes mostraram-se positivos em 5 deles. O segundo episódio, em Bauru, teve início em julho de 1990, tendo sido notificados 12 casos e 20 contactantes. "A dificuldade de identificação e tratamento dos envolvidos levou a SUCEN a tratar com cloroquina todos aqueles que tivessem feito uso em comum de seringas ou agulhas com os casos confirmados com seus contactantes, independentemente da existência ou não de sintomas sugestivos de malária ou do resultado dos exames hemoscópicos"3 (Tabela 1).

Os dois casos de malária congênita foram detectados no município de São Paulo, $P$. falciparum com êxito letal no quinto dia da doença, e no município de Ribeirão Preto, $P$. vivax com evolução benigna. Em ambos, as mães eram procedentes de áreas endêmicas de malária do país. Transmissão por acidente de trabalho $(2$ casos por $P$. vivax), envolveu pessoal de enfermagem de hospitais em São Paulo e São José dos Campos. O caso de malarioterapia por $\boldsymbol{P}$. vivax, foi decorrente de um paciente portador de atrofia muscular, devido a uma neurovirose. Após dois acessos febris foi instituída a terapêutica da malária.

A comparação de alguns aspectos clínicoepidemiológicos entre os pacientes infectados por 
Andrade JCR, Wanderley DMV. Malária induzida no Estado de São Paulo, Brasil. Revista da Sociedade Brasileira de Medicina Tropical 24:157-161, jul-sel, 1991

transfusão de sangue e os por agulha contaminada, avaliada na Tabela 2 , mostrou que a idade média - 22,6 anos desse último grupo é menor $(p<$ 0,005 ) que aqueles infectados por transfusão de sangue - 39,5 anos. Maior participação de indivíduos do sexo masculino no Grupo II do que no Grupo I, também foi observada. Embora o tempo médio decorrido entre o aparecimento dos sintomas e a realização do diagnóstico, com consequente início do tratamento tenha variado de 20,6 dias no Grupo I a 33,6 dias no Grupo II, estas diferenças não foram estatisticamente significantes ( $p>0,10$ ). Ressalta-se no Grupo l, um paciente que permaneceu 90 e outro 113 dias com sintomas sem que fosse feita a suspeita da malária transfusional, embora tivessem passado por vários tratamentos no período.

\section{DISCUSSÃO}

O primeiro caso de malária transfusional através do uso de sangue estocado data de $1941^{4}$. Embora cada vez mais seja dada atenção para a seleção de doadores e à preservação de sangue estocado, o grande uso de transfusões de sangue em medicina e cirurgia tem aumentado o número de casos de infeç̧ão induzida. Este aumento da incidência da malária induzida também pode estar relacionado com a melhoria do método diagnóstico, uma vez que a malária importada no Estado de São Paulo vem apresentando tendência decrescente a partir de $1988^{\circ}$. Neste aspecto, a vigilância epidemiológica deve garantir a agilidade do fluxo de notificação de modo a detectar e tratar precocemente as fontes de infecção importadas. A triagem adequada de doadores deve ser incrementada. Dispositivo legal sancionado em

Tabela 1 - Casos de malária induzida distribuidos por mecanismos de transmissão e espécie de plasmódio. Estado de São Paulo, 1983 a julho de 1990.

\begin{tabular}{|c|c|c|c|c|c|c|c|c|c|c|}
\hline \multirow{2}{*}{ Ano } & \multirow{2}{*}{$\begin{array}{c}\text { Total de } \\
\text { Casos } \\
\text { Notificados }\end{array}$} & \multirow{2}{*}{$\begin{array}{c}\text { Total de } \\
\text { Casos } \\
\text { Induzidos }\end{array}$} & \multicolumn{4}{|c|}{ Mecanismo de Transmissāo } & \multicolumn{4}{|c|}{ Espécie de Plasmódio } \\
\hline & & & $\begin{array}{c}\text { Transfu- } \\
\text { sional }\end{array}$ & "Agulha" & Congènita & Excepcional & P.vivax & P.falciparum & $P . v+P . f$ & P.malarie \\
\hline 1983 & 1666 & 2 & 1 & - & - & $1^{*}$ & 2 & - & - & - \\
\hline 1984 & 1885 & 12 & 9 & - & 2 & $1^{* *}$ & 4 & 2 & 5 & 1 \\
\hline 1985 & 2201 & 5 & 4 & - & - & $1^{* *}$ & 3 & 2 & - & - \\
\hline 1986 & 2686 & 4 & 4 & - & - & - & 2 & 2 & - & - \\
\hline 1987 & 3066 & 3 & 2 & 1 & - & - & 3 & - & - & - \\
\hline 1988 & 2499 & 2 & 1 & 1 & - & - & 2 & - & - & - \\
\hline 1989 & 2314 & 15 & 2 & 13 & - & - & 15 & - & - & - \\
\hline 1990 & 1123 & 15 & 2 & 13 & - & - & 15 & - & - & - \\
\hline Total & 17440 & 58 & 25 & 28 & 2 & 3 & 46 & 6 & 5 & 1 \\
\hline (\%) & & & $(43,1)$ & $(48,3)$ & $(3,4)$ & $(5,2)$ & $(79,4)$ & $(10,3)$ & $(8,6)$ & $(1,7)$ \\
\hline
\end{tabular}

* malarioterapia

* * acidente de trabalho 
Andrade JCR, Wanderley DMV. Malária induzida no Estado de São Paulo, Brasil. Revista da Sociedade Brasileirc de Medicina Tropical 24:157-161, jul-set, 1991

Tabela 2 - Aspectos clinico-epidemiológicos dos pacientes infectados através de transfusões de sangue e do uso de agulhas contaminadas. Estado de São Paulo, 1983 a julho de 1990.

\begin{tabular}{lcc}
\hline \multicolumn{1}{c}{$\begin{array}{c}\text { Aspectos } \\
\text { Clínico-epidemiológicos }\end{array}$} & $\begin{array}{c}\text { Grupo I } \\
\text { Transfusional }\end{array}$ & $\begin{array}{c}\text { Grupo II } \\
\text { " Agulha " }\end{array}$ \\
\hline Número casos & 25 & 28 \\
Idade média lanos) & 39,5 & 22,6 \\
Sexo (\%) & $28,0 \mathrm{~F}$ & $14,3 \mathrm{~F}$ \\
& $62,0 \mathrm{M}$ & $85,7 \mathrm{M}$ \\
Parasitemia média & + & + \\
Evolução & 16 bito & benigna \\
Sintomatologia & rica * & abundante ** \\
Tempo médio (dias) & & 33,6 \\
sintoma / diagnóstico & 20,6 & \\
\hline
\end{tabular}

* febre combinada com pelo menos 3 outros sintomas

* febre combinada com pelo menos 4 outros sintomas

1988, através de Lei 7649 de $25 / 01^{6}$, estabelece a obrigatoriedade do cadastramento dos doadores de sangue, bem como a realização de exames laboratoriais no sangue coletado com vistas a detectar hepatite B, sífilis, doença de Chagas, malária e AIDS, objetivando a prevenção da propagação de doenças. A exigência da feitura de testes de malária restringe-se às áreas endêmicas, definidas pelo Ministério da Saúde.

À luz das informações analisadas na presente revisão, no que se refere à transmissão transfusional, pode-se inferir que este aspecto da transmissão da malária esteja sob controle a curto prazo, haja visto a diminuição do número de casos notificados e a estruturação dos serviços de hemoterapia no Estado de São Paulo. No entanto no que toca à transmissão por "agulha" entre toxicômanos, a malária passa a se destacar. Considerando-se o longo período decorrido entre o aparecimento dos sintomas e o diagnóstico dos casos, 33,6 dias em média, e que os pacientes não se encontravam hospitalizados durante este tempo, portanto, fazendo uso de drogas em grupos, facilmente pode-se supor que seja grande o risco de disseminação por esta via, da malária em particular e de outras doenças em geral. Medida de intervenção direcionadas a este tipo de transmissão devem ser estruturadas pelos serviço de saúde.

\section{SUMMARY}

A review is presented of 58 induced malari cases diagnosed in the São Paulo State, from January 1983 to July 1990 . These cases represent $0.3 \%$ of th malaria notifications. The parameters sex, age parasitaemia, clinical symptoms, clinical course an mean time interval between disease onset and diagnosi were studied in 25 blood-transmitted cases and 2 related to sharing needles. Plasmodium vivax wa diagnosed in $79.4 \%$ and in $5.6 \%$ Plasmodium vivax an Plasmodium falciparum were seen. Lower mean ag ( $\mathrm{p}<0,005$ ) in intravenous drug users when compare in the blood-transmitted group were observed. Th former also had a mean time interval between diseas onset and diagnosis (33.6 days) and patients with blood transmitted disease ( 20.6 days). No statistical significan differences were observed $(p>0,10)$. Even though fe induced malaria cases have been diagnosed in $S a$ Paulo, State, and considering that blood-transmitte malaria is now under control, the occurrence of malar due to sharing needles among intravenous drug user should be regarded as a risk for the reintroduction of th disease in malaria - free areas of São Paulo State. 
Andrade JCR, Wanderley DMV. Malária induzida no Estado de São Paulo, Brasil. Revista da Sociedade Brasileira de Medicina Tropical 24:157-161, jul-set, 1991

Key-words: Malaria. Epidemiology. Transfusional malaria. Malaria among drugs users.

\section{AGRADECIMENTOS}

Os autores agradecem a Pesquisadora Científica da SUCEN, Maria Cecília Goi Porto Alves pela realização dos testes estatísticos.

\section{REFERÊNCIAS BIBLIOGRÁFICAS}

1. Andrade JCR, Anjos CFD, Wanderley DMV, Alves MJCP, Campos P. Foco de malária no Estado de São Paulo, Brasil. Revista de Saúde Pública 20: 323-326, 1986.

2. Barata LCB, Gutierrez EB, Dutra AP. Malária Transfusional: Descrição de 6 casos induzidos em doadores de sangue na Região Metropolitana de São Paulo. In: Resumos do XXV Congresso da Sociedade Brasileira de Medicina Tropical, Florianópolis p. 252, 1989.

3. Barata LCB, Andriguetti MTM, Cortaz MC, Meneguetti LC. Nota sobre surto de malária em usuários de drogas injetáveis no Município de São Paulo, Brasil. Revista de Saúde Pública 24: 321$322,1990$.

4. Bruce-Chwatt LJ. Transfusion malaria. Bulletin of the World Health Organization 50: 337-346, 1974.

5. Bruce-Chwatt LJ. Transfusion malaria revisited. Tropical Diseases Bulletin 79: 827-840, 1982.

6. Diário Oficial da União. Lei 7.649 de 25 de janeiro de 1988. Seção I p. 1.609, 1988.

7. Lo SS, Andrade JCR, Condino MLF, Alves MJCP, Semeghini MG, Galvão EC. Malária em usuários de drogas de administração endovenosa associada a soropositividade para HIV. Revista de Saúde Pública 25:17-22, 1991.

8. Secretaria de Estado da Saúde. Superintendência de Controle de Endemias (SUCEN). Relatório Anual de Avaliação do Programa de Controle de Malária no Estado de São Paulo, 1989.

9. Tauil PL. Malária: Agrava-se o quadro da doença no Brasil. Ciência Hoje 11: 58-64, 1984.

10. Wanderley DMV, Andrade JCR, Alves MJCP, Alves MCGP, Matos MR, Gurgel SM, Igreja RP. Malária no Estado de São Paulo: Avaliação de Aspectos da Vigilância Epidemiológica. Cadernos de Saúde Pública 5: 296-304, 1989.

11. Wanderley DMV, Andrade JCR, Meneguetti LC, Chinelatto MJ, Dutra AP. Malária no Estado de São Paulo, Brasil, 1980 - 1983. Revista de Saúde Pública 19:28-36, 1985. 